Received 00th January 20xx, Accepted 00th January 20xx DOI: $10.1039 / x 0 x x 00000 x$

\title{
Quadruplex DNA-Guided Ligand Selection from Dynamic Combinatorial Libraries of Acylhydrazones ${ }^{\dagger}$
}

\author{
Oksana Reznichenko, ${ }^{\mathrm{a}, \mathrm{b}}$ Anne Cucchiarini, ${ }^{\mathrm{a}, \mathrm{b}}$ Valérie Gabelica ${ }^{\mathrm{c}}$ and Anton Granzhan $*^{\mathrm{a}, \mathrm{b}}$ \\ Dynamic combinatorial libraries of acylhydrazones were prepared from diacylhydrazides and several cationic or neutral \\ aldehydes in the presence of 5-methoxyanthranilic acid catalyst. Pull-down experiments with magnetic beads \\ functionalized with a G-quadruplex (G4)-forming oligonucleotide led to the identification of putative ligands, which were \\ resynthesized or emulated by close structural analogues. G4-binding properties of novel derivatives were assessed by \\ fluorimetric titrations, mass spectrometry and thermal denaturation experiments, giving evidence of strong binding $\left(K_{\mathrm{d}}<\right.$ \\ 10 \\ $\mathrm{nM})$ \\ for \\ two \\ compounds.
}

\section{Introduction}

G-quadruplex (G4) structures of DNA and RNA are involved in numerous cellular processes such as DNA replication, transcription, telomere maintenance, and RNA translation. ${ }^{1,2}$ Targeting of these structures with small molecules (ligands) paves a way for therapeutic control of biological processes that involve G4-mediated regulation..$^{3-5}$ However, considering the large number of putative G4-forming sequences in the human genome (over 700000 ), ${ }^{6}$ G4 ligands need to acquire selectivity to certain G4 structures over the others, in order to minimize possible off-target effects. Despite more than 20 years of active research, only moderate progress has been achieved in terms of intra-G4 selectivity of ligands. ${ }^{5,7,8}$ Clearly, the conventional strategies that rely on the synthesis of small libraries of putative ligands and subsequent assessment of their binding to a panel of G4 structures are insufficient to generate ligands with better affinity and selectivity, and novel approaches allowing tailor-made ligand design are urgently needed. Several strategies have been explored towards this end, including fragment-based screening, ${ }^{9,10}$ small-molecule microarrays, ${ }^{11}$ kinetic target-guided synthesis, ${ }^{12}$ and peptide libraries, ${ }^{13,14}$ resulting in identifications of novel G4 ligands with intriguing properties.

Dynamic combinatorial chemistry (DCC) is a supramolecular approach that exploits reversible chemical

\footnotetext{
a. CNRS UMR9187, Inserm U1196, Institut Curie, PSL Research University, 91405 Orsay, France.E-mail: anton.granzhan@curie.fr

b. CNRS UMR9187, Inserm U1196, Université Paris Saclay, 91405 Orsay, France.

c. Univ. Bordeaux, CNRS, INSERM, ARNA, UMR 5320, U1212, IECB, 33600 Pessac, France.

+ Electronic Supplementary Information (ESI) available. See DOI: 10.1039/x0xx00000x
}

reactions to generate dynamic combinatorial libraries (DCL) of products under thermodynamic control. ${ }^{15,16}$ This method is particularly well-suited for the discovery of ligands and receptors, since the addition of an external species (a "target", or a "template") shifts the dynamic equilibrium towards the formation of the product(s) having the highest affinity to the target. ${ }^{17}$ While DCC has now been well established for protein targeting ${ }^{18,19}$ its application in the field of nucleic acids is much more restricted. ${ }^{20,21}$ Along these lines, Balasubramanian $^{22-24}$ and Ulven ${ }^{25}$ labs exploited DCC for the discovery (or optimization) of G4-DNA ligands; of note, all these approaches relied on the reaction of disulfide exchange and involved only small number of components, typically a central scaffold possessing a G4 affinity and a set of side chains. More recently, Dash et al. utilized a DCC approach based on the reversible formation of imines followed by pulldown and reduction, to identify novel carbazole-based G4DNA ligands. ${ }^{26}$ However, a serious drawback of imine-based DCC is that it requires the conversion of imines, identified from DCL analysis, into stable amine analogues whose affinity and selectivity to the target may be significantly different. Therefore, there is a need for the development of alternative chemistries suitable for nucleic acid-targeted DCC.

In this context, we have recently described cationic bis(acylhydrazones), such as $\mathrm{PyDH} 2$ and PhenDH2 (Chart 1), ${ }^{27}$ as novel, biologically active G4 ligands based on the wellestablished bis- 

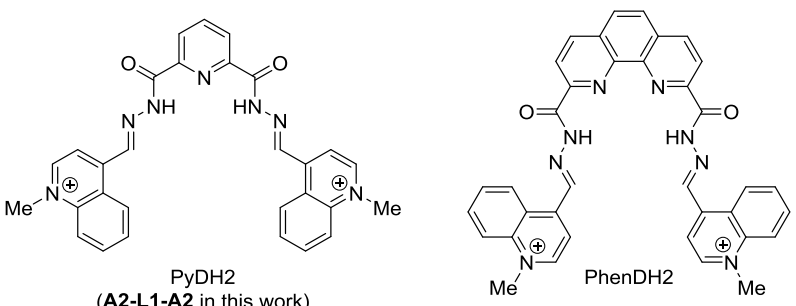

Chart 1 Structures of previously described cationic bis(acylhydrazones).

quinolinium scaffold. ${ }^{28,29}$ An interesting property of $\mathrm{N}$-acylhydrazones is their reversible formation in the presence of suitable nucleophilic catalysts. ${ }^{30,31}$ Consequently, acylhydrazone exchange has been extensively utilized in DCC approaches targeting proteins. ${ }^{18,32}$ At the same time, this reaction has barely been exploited for nucleic acid-targeting DCC, except for a few noteworthy cases dealing with nucleic acid delivery applications. ${ }^{33,34}$ Building on these premises, in this work we investigated the suitability and the pitfalls of the acylhydrazone-based DCC for the identification of G4 ligands as well as G4-DNA binding properties of the resulting compounds.

\section{Results and Discussion}

To access the feasibility of synthesis of bis(acylhydrazones) in the conditions compatible with native structures of G4-DNA, we initially studied the reaction of equimolar amounts of the cationic aldehyde $\mathbf{A 1} \neq$ with pyridine-2,6-dihydrazide $\mathbf{L} \mathbf{1}$ in the aqueous DCC buffer ( $100 \mathrm{mM} \mathrm{NH}_{4} \mathrm{OAc}, 1.5 \mathrm{mM} \mathrm{KCl}$, $\mathrm{pH}$ 6.4) in the presence of four anthranilic acid derivatives as nucleophilic catalysts (Fig. 1, a). HPLC analysis of reaction mixtures (Fig. 1, b) demonstrated that in presence of 5-methoxyantranilic acid (1b) the reaction almost reached equilibrium after $24 \mathrm{~h}$, whereas 1a and 1c were less efficient, and only traces of products were detected when the reaction was performed in the presence of $\mathbf{1} \mathbf{d}$. Therefore, $\mathbf{1} \mathbf{b}$ was employed as catalyst in the subsequent DCC experiments.

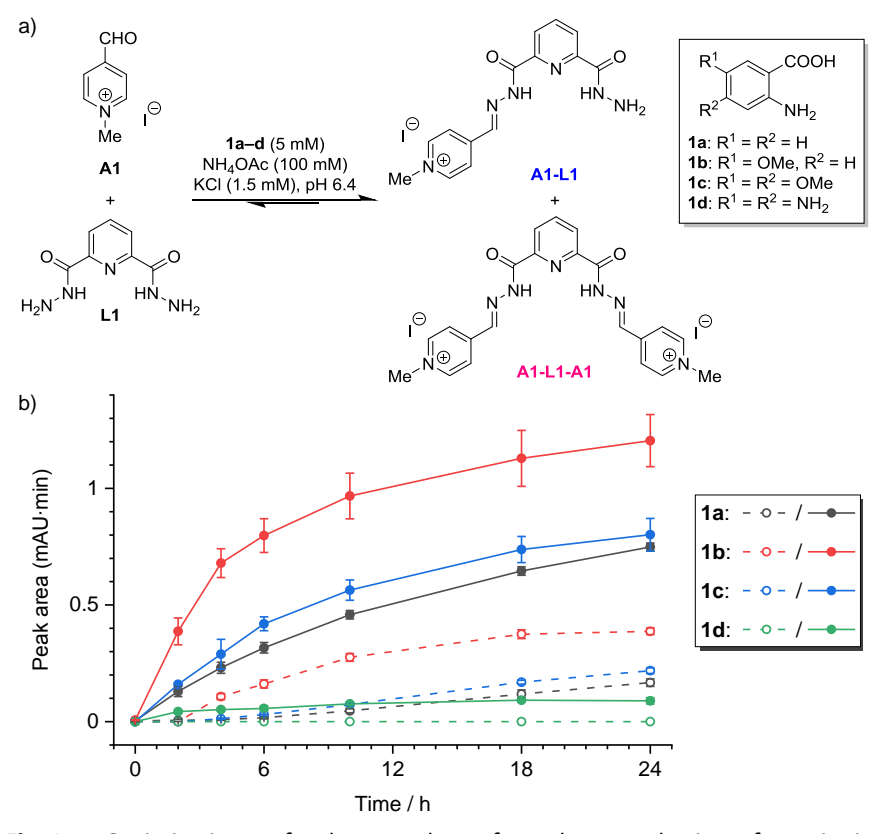

Fig. 1 Optimization of the catalyst for the synthesis of cationic bis(acylhydrazones). a) Reaction scheme; $¥$ b) time course of formation of A1-L1 (empty circles, dashed lines) and A1-L1-A1 (filled circles, solid lines) upon the reaction of $\mathbf{A} \mathbf{1}$ and $\mathbf{L 1}$ ( $40 \mu \mathrm{M}$ each) in DCC buffer in the presence of catalysts 1ad $(5 \mathrm{mM})$. Data from three independent experiments.

Next, we generated a first combinatorial library (DCL1) through the reaction of two aldehydes (A1 and $\mathbf{A 2}$ ) and three dihydrazides (L1-L3). The employed conditions (10 mM of $\mathbf{1 b}$, incubation time of $24 \mathrm{~h}$ ) resulted in significant conversion of the reagents (Fig. 2, a) and led to the formation of 15 products, including six mono-acylhydrazones and nine bis(acylhydrazones, all of which could be successfully separated by HPLC (Fig. 2, b); the peaks were assigned by using pairwise combinations of reagents. Of note, all six symmetric bis(acylhydrazones) present in this library (pink in Fig. 2) were synthesized in the preparative fashion in our previous work and their G4-binding properties were documented, ${ }^{27}$ enabling a subsequent validation of the G4-templated DCC approach. 


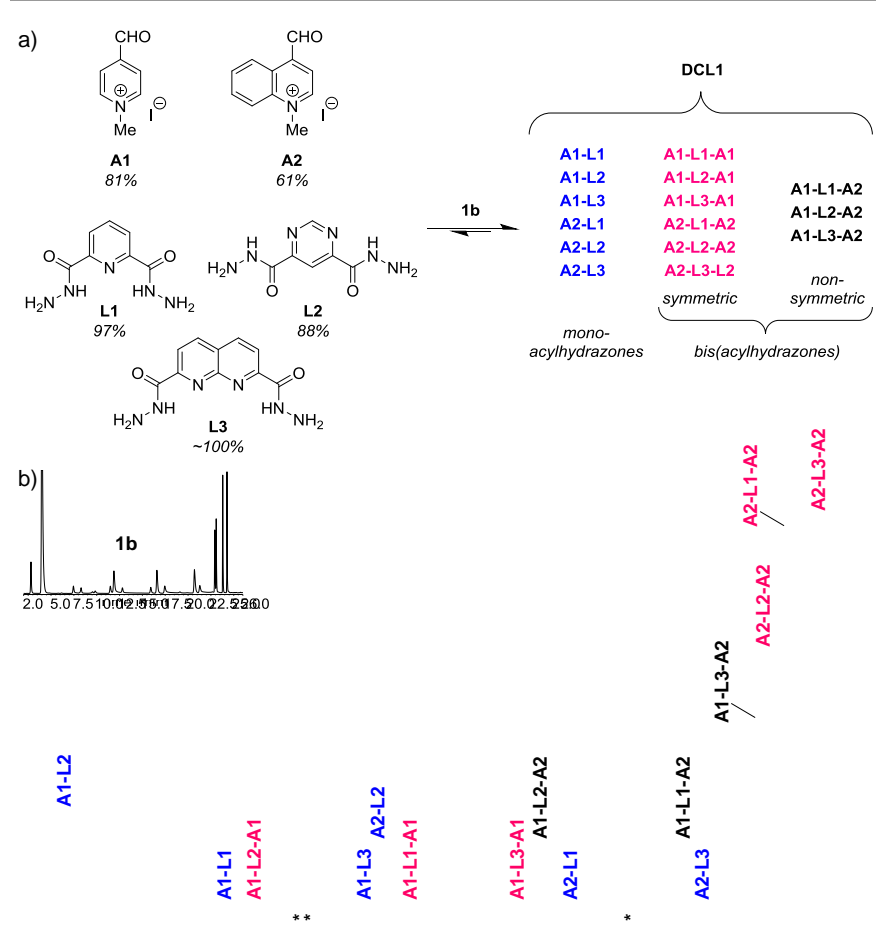

Fig. 2 a) Schematic representation and b) HPLC profile of a 15-membered DCL1 obtained upon 24-h reaction of two cationic aldehydes with three dihydrazides. $₹ c$ (A1) $=c(\mathbf{A} \mathbf{2})=120 \mu \mathrm{M}, c(\mathbf{L} \mathbf{1})=c(\mathbf{L} \mathbf{2})=c(\mathbf{L} \mathbf{3})=40 \mu \mathrm{M}, c(\mathbf{1} \mathbf{b})=10 \mathrm{mM}$ in $100 \mathrm{mM} \mathrm{NH}_{4} \mathrm{OAc}, 1$ $\mathrm{mM} \mathrm{KCl}$ buffer, $\mathrm{pH}$ 6.0. Italicized numbers in panel a) indicate the conversion of the reagents in the "blank" library. Peaks labelled with asterisks in b) could not be assigned.

One advantage of the acylhydrazone-based DCC is the stability of the products in the conditions of HPLC analysis, avoiding the need of the "freezing" step such as the borohydride reduction in imine-based DCC. ${ }^{26}$ Thus, proteintemplated DCLs of acylhydrazones are typically analysed by direct HPLC injection: in HPLC conditions, the protein template denatures and bound ligands are instantly released, allowing their quantification and comparison with the "blank" library obtained in the absence of the template. ${ }^{32,35,36}$ To verify the applicability of this method to G4-templated DCLs, we performed HPLC injections of equimolar mixtures ( $25 \mu \mathrm{M}$ each) of pure, extemporarily prepared A2-L1-A2 (i.e., PyDH2, a known strong G4-DNA binder) and A1-L2-A1 (a very poor G4-DNA binder) ${ }^{27}$ in the absence and in the presence of $50 \mu \mathrm{M}$ of a G4DNA oligonucleotide (Pu24T, sequence: Table S1+). The presence of G4-DNA had no effect on A1-L2-A1 that was eluted in a sharp peak. However, the peak of A2-L1-A2 was shifted and strongly distorted in the presence of G4-DNA (Fig. $\mathrm{S} 1+)$. A possible explanation of this phenomenon is that the complex of G4-DNA with strong binders is not instantly dissociated, but retained in the column head after HPLC injection, leading to a gradual dissociation occurring during the HPLC run and manifested as peak tailing. Indeed, G4-DNA structures were shown to be extremely stable in a range of non-physiological conditions, including up to $50 \%$ acetonitrile; ${ }^{37,38}$ moreover, the dissociation constants of best G4-DNA binders (including A2-L1-A2) are typically found in the low-nanomolar range, i.e., one to three orders of magnitude lower than that of typical protein inhibitors discovered by protein-templated $\mathrm{DCC},{ }^{35,36}$ and this strong binding can lead to additional stabilization of the folded form of G4-DNA. We attempted to release bound ligands by adding an excess ( 5 molar equivalents) of PhenDC3, one of the most potent and well-characterised ligands described to date, ${ }^{29}$ as a competitor prior to HPLC analysis, ${ }^{39}$ or by supplementing the samples with equal volume of formamide. However, in either case the original peak shape could not be restored, making peak integration unreliable. These observations implied that the comparative approach using the direct HPLC analysis could not be employed for reliable assessment of G4-DNA-templated libraries. ${ }^{16}$

To overcome this issue, we turned to the "capturing" protocol that uses biotinylated G4-DNA targets captured by streptavidin-coated paramagnetic beads. Instead of comparing templated and non-templated libraries, this approach seeks to identify the best binders pulled-down by DNA-covered beads from "frozen" libraries, following ligand release in more harsh conditions. ${ }^{16}$ The principle of this method is shown on Fig. 3, a. To validate this approach, DCL1 was incubated in the presence of 5'-biotinylated Pu24T. The structure of this G4-DNA, formed by a modified sequence from the NHE III $_{1}$ element of $M Y C$ promoter, was thoroughly characterized and comprises several distinct structural elements (an accessible 5'-tetrad, different grooves, double-chain-reversal loops and a "snap-back" diagonal loop covering the 3 '-tetrad) which may serve as potential binding sites for small-molecule ligands. ${ }^{40,41}$ In parallel, we employed two biotinylated, non-G4 controls of comparable molecular size, namely a single-stranded oligonucleotide $\left(d T_{22}\right)$ and an 18-mer hairpin ( $h p 2$, Table $\mathrm{S} 1+$ ) containing a non-nucleoside (i.e., hexaethyleneglycol) loop, introduced to avoid the possible binding of ligands in the loop region. Following a 24-h incubation, the reaction was arrested by increase of $\mathrm{pH}$ and pre-washed magnetic beads were added, resulting in pull-down of DNA-ligand complexes. After a thorough washing, the pulled-down ligands were released from the beads by addition of neat formamide and heating to $50{ }^{\circ} \mathrm{C}$, and analysed by HPLC; the resulting peak areas were normalized with respect to control (untreated) library to account for the differences in initial composition and the differences in UV absorption by ligands, and compared with a sample treated with magnetic beads in the absence of biotinylated DNA, to account for non-specific ligand absorption on the beads. The results (Fig. 3, b) demonstrated that five ligands were selectively pulled down from the library by Pu24T-covered beads, in the following relative order: A2-L3-A2 > A2-L1-A2 > A1-L3-A2 $\approx$ A2-L2-A2 > A1-L1-A2. Satisfyingly, this ranking was in a good 
a)
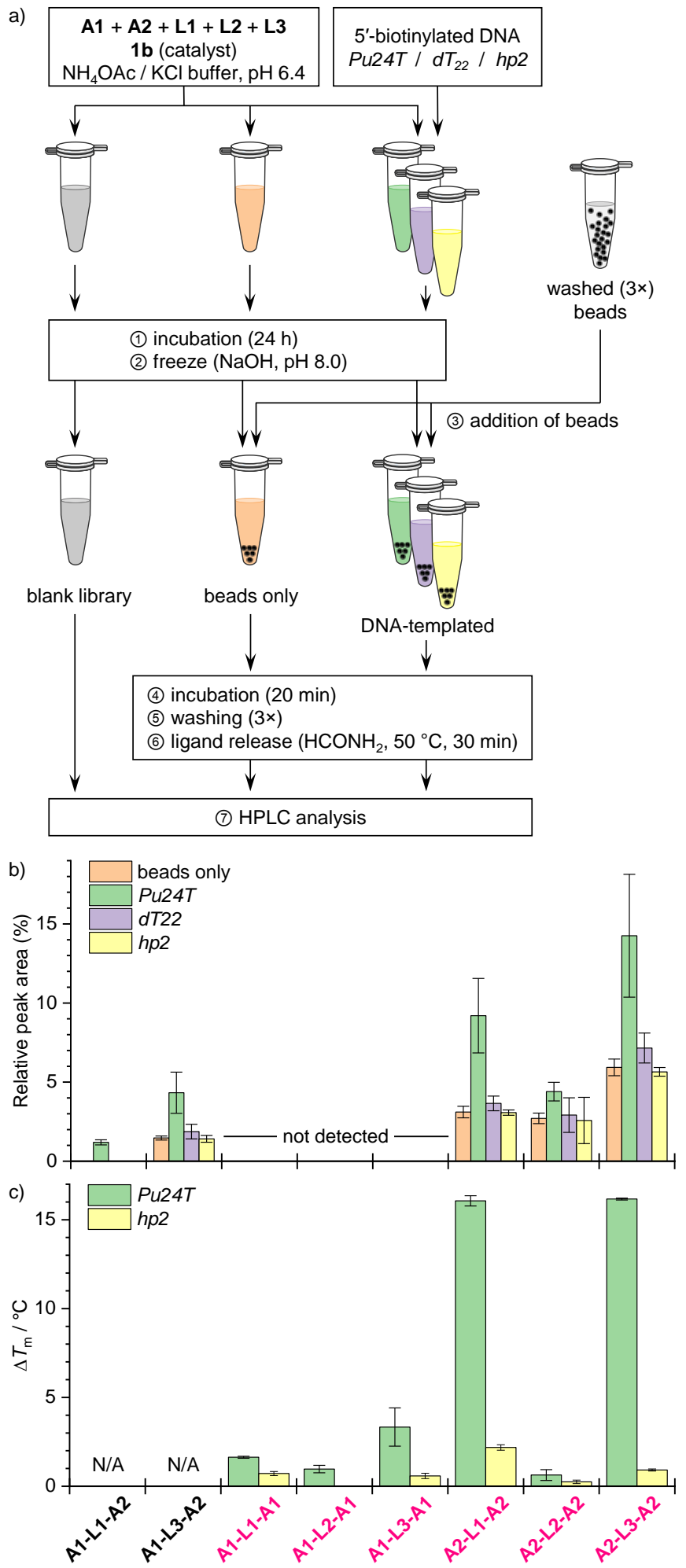

Fig. 3 a) Experimental protocol for DNA-targeted DCC (here: DCL1) using biotinylated DNA oligonucleotides and streptavidin-coated magnetic beads. b) Relative (with respect to the "blank library") amounts of components of DCL1 released after pull-down with naked beads or biotinylated oligonucleotides; data are mean \pm s.d. from three independent experiments. c) Ligand-induced stabilization of G4-DNA Pu24T and hairpin $h p 2(0.2 \mu \mathrm{M})$ in fluorescence-melting experiments performed with pure, extemporarily synthesized symmetric components of DCL1 $(1.0 \mu \mathrm{M})$. Data are mean \pm s.d. from three measurements. $\mathrm{N} / \mathrm{A}=$ not available. agreement with the ligand-induced stabilization of Pu24T observed in fluorescence-melting experiments performed with pure samples of symmetric ligands that demonstrated that A2L3-A2 and A2-L1-A2, but not A2-L2-A2, were good G4 binders (Fig. 3, c). Of note, non-specific binding of ligands to magnetic beads was not negligible in these experiments, as evidenced by the amount of ligands pulled-down in the absence of biotinylated DNA ("beads only"); at the same time, no ligand was selectively pulled-down in the presence of single-stranded or hairpin DNA, demonstrating the absence of binding to nonG4 DNA targets. This fact also agrees with the results of fluorescence-melting experiments that demonstrate minimal effect of all ligands on thermal stability of the hairpin hp2 (Fig. $3, \mathrm{c}$.

Inspired by these results, we designed two other libraries: a 14-membered $D C L 2$, prepared from the dihydrazide L1, cationic aldehyde $\mathbf{A} \mathbf{2}$ and its isomer $\mathbf{A} \mathbf{3}$, as well as two novel aldehydes (A4 and A5), and an 18-membered DCL3 was constructed from L1, its 4-(2-morpholinoethoxy) derivative L4, and aldehydes A2, A3 and A5 (Scheme 1; Table S2t and Fig. $\mathrm{S} 2+$ ). In both cases, we introduced the fragments that were uncharged (A4) or partially charged in the assay conditions (A5) in a hope to obtain ligands displaying a G4 affinity comparable to that of the prototype bis(acylhydrazones) (Chart 1) but a lower permanent cationic charge. Of note, the conversion of aldehyde $\mathbf{A 5}$ in the conditions of DCL synthesis was significantly lower (34-41\%) comparing with heterocyclic aldehydes A2-A4 (80-100\%), presumably due to its lower reactivity owing to the presence of an electron-donating amine substituent in the benzene ring (Table S2). Nevertheless, acylhydrazone products containing the $\mathbf{A 5}$ fragment could be clearly detected in the resulting libraries (Fig. S2+). The pulldown experiments, performed with magnetic beads in the conditions identical to those presented in Fig. 3, demonstrated that, in the case of DCL2, A2-L1-A5 was the only compound massively and selectively extracted in the presence of Pu24T; even in this case, significant amounts of this ligand were detected in samples treated with $d T_{22}$ and $h p 2$ oligonucleotides (Fig. 4, a). Interestingly, A2-L1-A2, a good G4-DNA binder, was not selected from this library. In contrast, in the case of DCL3, a number of compounds were selectively extracted in the presence of Pu24T, in the following order: A2L1-A5 $\approx$ A2-L4-A5 > A2-L1-A2 $\approx$ A2-L4-A2 > A2-L1-A3 $\approx$ A2-L4A3 (Fig. 4, b). These compounds (in addition to A2-L1-A2 already described above) could be considered as promising G4-DNA binders, and we next sought to synthesize some of them in preparative fashion in order to confirm their G4binding properties.

The non-symmetric bis(acylhydrazone) A2-L1-A5, selected from both DCL2 and DCL3, was considered as a priority target. An initial attempt to synthesize this compound was undertaken through a "one-pot" reaction performed by heating of $\mathbf{L} 1$ with equimolar amounts of $\mathbf{A} 2$ and $\mathbf{A 5}$ in DMF, followed by chromatographic separation of the products. However, while mass-spectrometric analysis showed that three compound (A2-L1-A2, A2-L1-A5 and A5-L1-A5) formed in this reaction, they co-eluted on a preparative RP-HPLC column, 
rendering their separation impossible on a preparative scale. Therefore, we designed hybrid ligands $\mathbf{5 a}$ and $\mathbf{5 b}$, in which $\mathbf{N}$ methylquinolinium and 4-(4-methylpiperazino)phenyl moieties were linked to the central 2,6-pyridyl unit through two different combinations of carboxamide and acylhydrazone bonds. Indeed, we have previously demonstrated that G4binding properties of acylhydrazone ligands were nearly identical to the ones of their carboxamide counterparts ${ }^{27}$ and could reasonably expect that these hybrids emulate the G4binding properties of the non-symmetric bis(acylhydrazone) A2-L1-A5.

The synthesis of $\mathbf{5 a}$ and $\mathbf{5 b}$ is presented on Scheme 2. Both isomers were prepared from the commercially available monomethyl pyridine-2,6-dicarboxylate through amidation with the

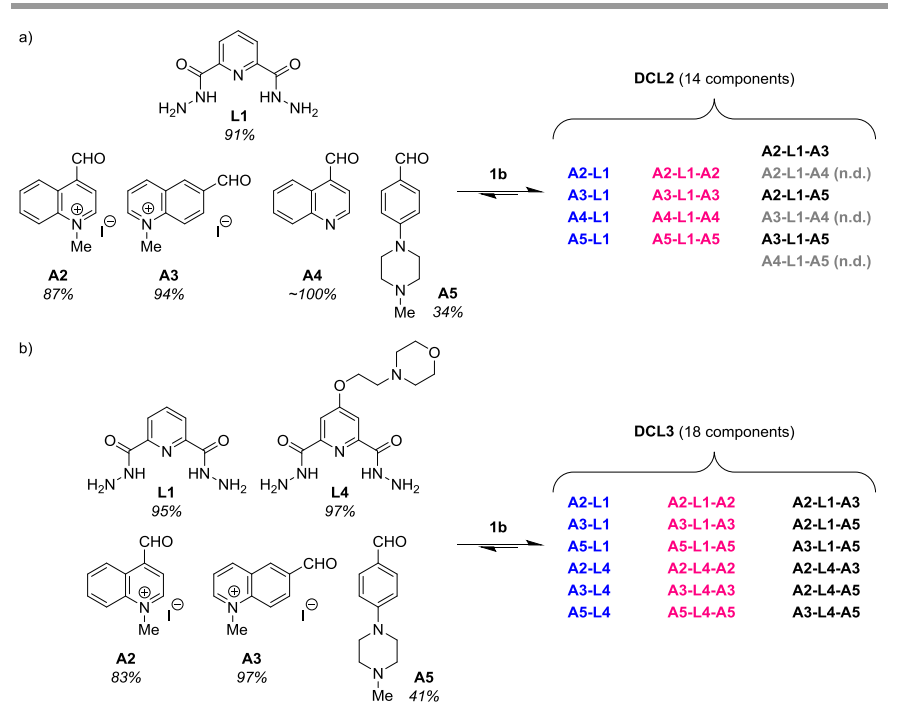

Scheme 1. Design of a) 14-component DCL2 and b) 18-component DCL3.‡ N.d. = components not detected in the blank library. Italicized numbers indicate the conversion of the reagents in "blank" libraries. The corresponding chromatograms are shown on Fig. S2+.
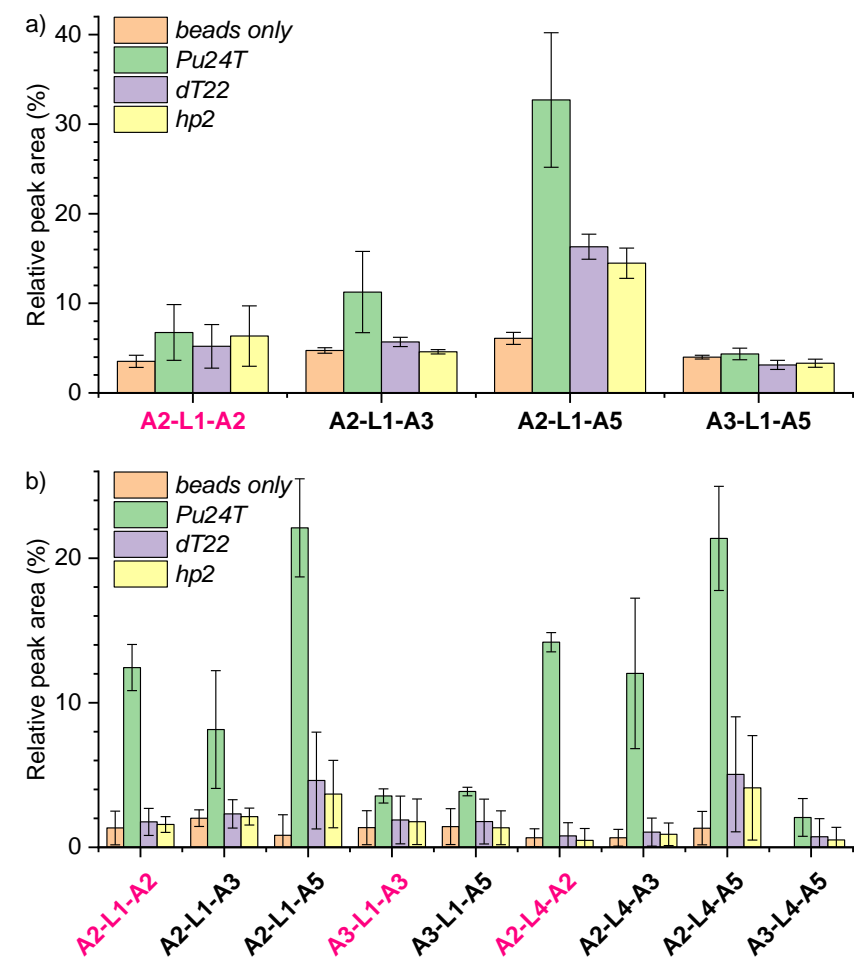

Fig. 4 Relative (with respect to the "blank library") amounts of components of a) DCL2 and b) DCL3, released after pull-down with naked beads or biotinylated oligonucleotides; data are mean \pm s.d. from three independent experiments.

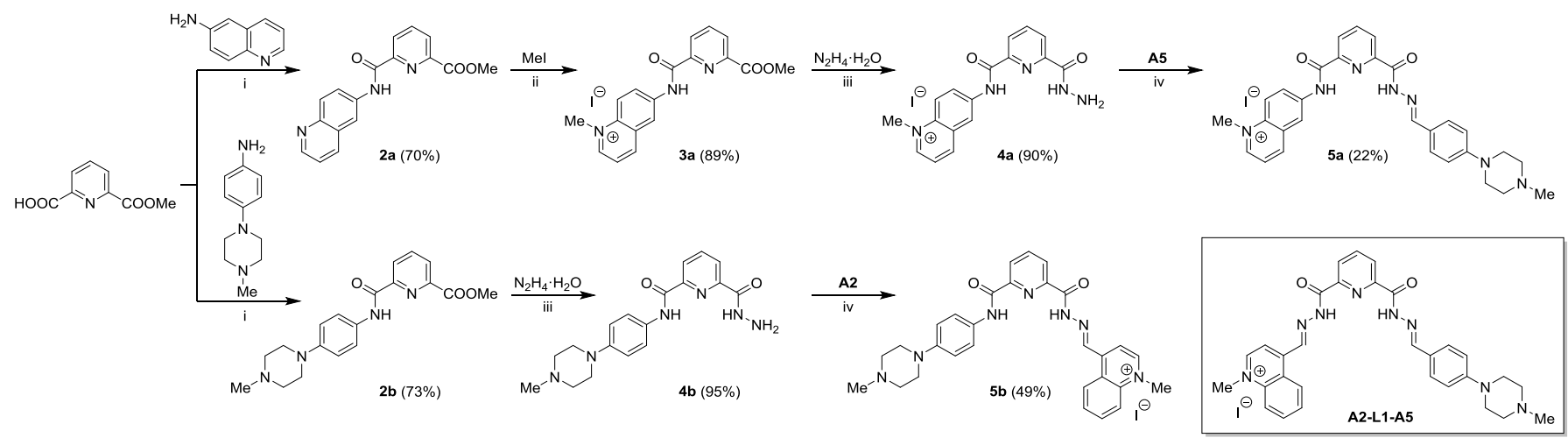

Scheme 2. Synthesis of non-symmetric (carboxamide/acylhydrazone) ligands 5 a and $\mathbf{5 b}$. Conditions: i) $\mathrm{EDCl} \cdot \mathrm{HCl}, \mathrm{HOBt}, \mathrm{DCM} / \mathrm{DMF}\left(10: 1 \mathrm{v} / \mathrm{v}\right.$ ), r.t., $18 \mathrm{~h}$; ii) $\mathrm{Me}{ }_{2} \mathrm{CO}$, reflux, 18 h; iii) $\mathrm{MeOH}$, room temp., 3 h; iv) $\mathrm{PrOH}, \mathrm{AcOH}$ cat., reflux, $18 \mathrm{~h}$. The structure of elusive A1-L1-A5 is shown for comparison.

corresponding aromatic amines to give the amides $\mathbf{2} \mathbf{a}-\mathbf{b}$, followed by methylation of the quinoline substituent yielding the salt 3a, hydrazinolysis of methyl esters to give the acylhydrazides $\mathbf{4 a}$ and $\mathbf{4} \mathbf{b}$, and the reaction of the latter with aldehydes $\mathbf{A} \mathbf{5}$ or $\mathbf{A 2}$, respectively. In addition, symmetric bis(acylhydrazones) A2-L4-A2 (selected from DCL3) and A5-L1A5 (not selected either from DCL2 or from DCL3, and chosen as a negative control) were prepared by straightforward condensation of the corresponding building blocks (ESI $\left.{ }^{\dagger}\right)$.

G4-binding properties of novel derivatives were initially studied by isothermal fluorimetric titrations that harness the ligand-induced fluorescence quenching of 5'-Cy5-labelled oligonucleotides. ${ }^{42}$ Performed at a constant temperature, this method is suitable to characterise of strong binders with $K_{\mathrm{d}}$ values down to few $\mathrm{nM}$, and is gaining increasing popularity in 
characterizing ligand-G4 interactions. ${ }^{13,43}$ The results of titrations, performed with four fluorophore-labelled G4-DNA oligonucleotides belonging to different folding topology groups (Pu24T and myc22: parallel, 25TAG: hybrid, 22CTA: antiparallel, sequences: cf. Table $\mathrm{S}^{\dagger}+$ ), confirmed the G4binding properties of novel derivatives (Table 1, titration curves: Fig. S3+). Compounds A2-L1-A2 and A2-L4-A2 had very similar and very high affinity to all four G-quadruplexes, with $K_{\mathrm{d}}$ values of 6 to $16 \mathrm{nM}$, which is comparable to that of the benchmark ligand PhenDC ${ }_{3}$ ( $K_{\mathrm{d}}$ of 2.4 to about $10 \mathrm{nM}$, as per $\mathrm{SPR}^{44}$ or Cy5-quenching titrations), ${ }^{42}$ and largely supersedes the affinity of several recently reported G4-binding peptides (determined by the same method). ${ }^{13,14}$ Hybrids $\mathbf{5 a}$ and $\mathbf{5 b}$ were significantly less active (with $K_{\mathrm{d}} \geq 90 \mathrm{nM}$ ), with $\mathbf{5 a}$ generally having lower affinity than $\mathbf{5 b}$. In particular, the interaction of 5a with telomeric G4-DNA (25TAG) was characterized by a $K_{\mathrm{d}}$ value of $2.9 \mu \mathrm{M}$, more than ten-fold larger than in case of parallel quadruplexes Pu24T and myc22 ( $K_{\mathrm{d}} \approx 250$ and $200 \mathrm{nM}$, respectively). Compound A5-L1-A5 did not quench the fluorescence of Cy5-labelled G4 substrates, a fact that can be interpreted either as poor G4 binding of this compound, or as unfavourable electron transfer with the fluorophore, resulting in the lack of quenching effect upon binding. Finally, none of ligands showed quenching of fluorophore-labelled hairpin $h p 2$ and single-stranded control $d T_{22}$ at concentrations lower than $1 \mu \mathrm{M}$ (Fig. S3+), giving evidence of

Table 1 Dissociation constants $\left(K_{\mathrm{d}} / \mathrm{nM}\right)$ of ligands to G4-DNA and a hairpin control ( $h p 2)$, determined from fluorimetric titrations with $5^{\prime}$-Cy5-labelled oligonucleotides. ${ }^{a}$

\begin{tabular}{lllll} 
Ligand & Pu24T & myc22 & 25TAG & 22CTA \\
\hline A2-L1-A2 & $7.6 \pm 0.6$ & $7.8 \pm 0.3$ & $11.3 \pm 0.5$ & $8.3 \pm 0.3$ \\
A2-L4-A2 & $11.8 \pm 1.8$ & $6.4 \pm 0.3$ & $16.1 \pm 0.8$ & $8.1 \pm 0.6$ \\
5a & $248 \pm 10$ & $199 \pm 7$ & $2890 \pm 140$ & $651 \pm 56$ \\
5b & $88 \pm 4$ & $131 \pm 9$ & $92 \pm 3$ & $313 \pm 27$ \\
A5-L1-A5 & $>10^{4}$ & $>10^{4}$ & $>10^{4}$ & $>10^{4}$
\end{tabular}

${ }^{a}$ Conditions: $c($ DNA $)=2 \mathrm{nM}, c($ ligand $)=0$ to $10 \mu \mathrm{M}$ in $10 \mathrm{mM} \mathrm{LiAsO}_{2} \mathrm{Me}_{2}, 100 \mathrm{mM}$ $\mathrm{KCl}, 0.5 \mathrm{w} / \mathrm{v} \%$ CHAPS, $0.05 \mathrm{v} / \mathrm{v} \%$ Triton X-100 buffer, $\mathrm{pH} 7.2 ; \lambda_{\mathrm{ex}}=590 \mathrm{~nm}, \lambda_{\mathrm{em}}=$ $675 \mathrm{~nm} . K_{\mathrm{d}}$ values are determined by numeric fitting of the data (means from three technical replicates) to a 1:1 binding model, with errors representing the standard error of the fitting parameter.

the absence of non-specific (i.e., unrelated to G4-DNA binding) quenching effect of ligands on the Cy5 probe.

Additional information about G4 binding of novel derivatives was obtained from native mass spectra of G4ligand complexes ${ }^{45}$ that were performed with two G4-DNA (PU24T and 25TAG). In all cases, the peaks of G4-ligand complexes could be clearly observed. Interestingly, mass spectra of 25TAG in the presence of A2-L1-A2 and A2-L4-A2 were largely dominated by the peaks of $1: 1$ complexes, formed upon ejection of one of the two $\mathrm{K}^{+}$ions initially present in this substrate (Fig. S4, $\mathrm{ESI}^{+}$). Ejection of $\mathrm{K}^{+}$cations was previously observed with other ligands of the bis(quinolinium) family, and attributed to the ligand-induced change from hybrid to antiparallel conformation of this G4-DNA. ${ }^{46}$ In contrast, 1:1 complexes of 25TAG with hybrids $\mathbf{5 a}$ and $\mathbf{5 b}$ showed the peaks attributed to both hybrid $\left(2 \mathrm{~K}^{+}\right)$and anti-parallel $\left(1 \mathrm{~K}^{+}\right)$species (Fig. S4, ESIt). This observation, along with the additional signals of 2:1 (ligand: G4) complexes, suggests their multiple binding modes to the 25TAG quadruplex. In the case of Pu24T, no $\mathrm{K}^{+}$ejection was observed, and only minor peaks of 2:1 complexes could be detected with all ligands, consistent with the presence of a single ligand-accessible binding site (i.e., the 5 '-tetrad). ${ }^{40}$ The relative intensities of the peaks of the ligandbound and free G4-DNA were used for the calculation of apparent binding constants (Table $\mathrm{S}^{+}{ }^{+}$). These values were generally found in a relatively good agreement with the results of fluorimetric titrations, except for the interaction of 5a with telomeric 25TAG that was characterized by a $K_{d}$ value of $0.33 \mu \mathrm{M}$, almost 10 -fold lower than the value obtained by fluorimetry $(2.89 \mu \mathrm{M}$, cf. Table 1$)$. This discrepancy may be due to a particular binding mode of this ligand, unfavourable for interaction with the fluorophore, such as binding in the vicinity of the unlabelled $3^{\prime}$-tetrad. ${ }^{42}$

Finally, DNA binding of ligands was assessed by fluorescence-melting experiments, based on thermal denaturation of double-labelled sequences. ${ }^{47}$ The conditions of these experiments, namely the $\mathrm{K}^{+}$content of the buffers, were selected so that the four G4-DNA substrates and the hairpin hp2 denature at comparable temperature in the absence of ligands $\left(T_{\mathrm{m}}{ }^{0} \approx 60^{\circ} \mathrm{C}\right)$, thus allowing direct comparison of ligandinduced thermal shifts $\left(\Delta T_{\mathrm{m}}\right)$. The results (Fig. 5) show that, in all cases, no preferential stabilization of PU24T over three other G4-DNA substrates was observed; instead, most compounds preferentially stabilized the 25TAG quadruplex, with $\Delta T_{\mathrm{m}}$ values of up to $30{ }^{\circ} \mathrm{C}$ (A2-L1-A2). Consistently with the results of fluorimetric titrations and mass-spectrometry experiments, hybrids $\mathbf{5 a}$ and $\mathbf{5} \mathbf{b}$ induced lower stabilization of most substrates compared with A2-L1-A2, but higher than A5L1-A5. However, in the case of antiparallel quadruplex 22CTA, compounds 5a $\left(\Delta T_{\mathrm{m}}=15.3^{\circ} \mathrm{C}\right)$ and, particularly, 5b $\left(\Delta T_{\mathrm{m}}=\right.$ $\left.18.8^{\circ} \mathrm{C}\right)$ were almost as efficient as A2-L1-A2 $\left(\Delta T_{\mathrm{m}}=22.2^{\circ} \mathrm{C}\right)$ and A2-L4-A2 $\left(\Delta T_{\mathrm{m}}=17.7^{\circ} \mathrm{C}\right)$ despite large differences in binding constants (Table 1). Conversely, compound A2-L4-A2 systematically induced less efficient stabilization of all G4-DNA substrates compared with the parent 

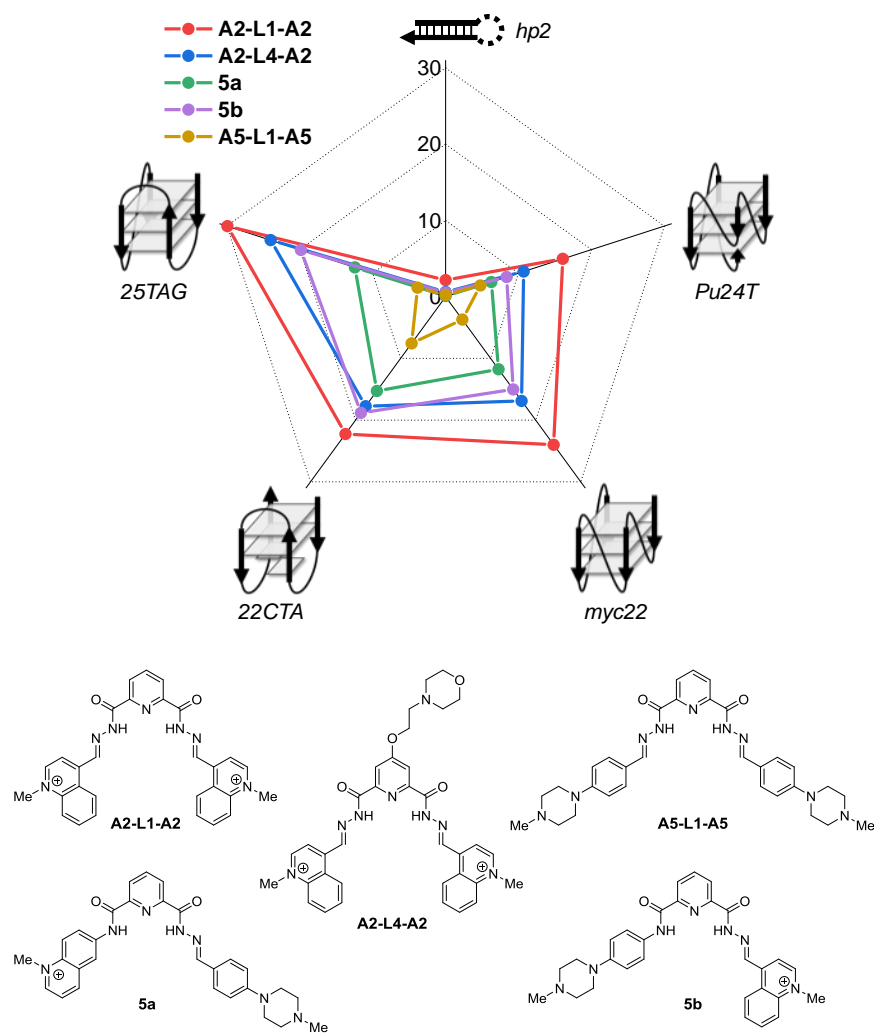

Fig. 5 Ligand-induced stabilization $\left(\Delta T_{\mathrm{m}},{ }^{\circ} \mathrm{C}\right.$ ) of G4-DNA (Pu24T, myc22, 22CTA 25TAG) and hairpin control ( $h p 2$ ) observed in fluorescence-melting experiments performed with compounds A2-L1-A2, A2-L4-A2, 5a, 5b, and A5-L1-A5 [c(DNA) = $0.2 \mu \mathrm{M}, c$ (ligand) $=1.0 \mu \mathrm{M}$, buffer composition: cf. Experimental Part $\left.{ }^{\dagger}\right]$. Data are mean values from three measurements. The structures of DNA substrates are schematically shown next to each axis and the structures of compounds below the graph.

ligand A2-L1-A2, although both compounds were pulled down from DCL3 at almost identical levels (Fig. 4, b) and displayed very close $K_{\mathrm{d}}$ values in the fluorimetric assay (Table 1 ). These differences between $K_{\mathrm{d}}$ values and thermal stabilization effects may be due to their different binding mode; indeed, ligands with large entropic contributions to the binding yield higher thermal stabilization effects. ${ }^{48}$ Of note, fluorescence melting experiments confirmed the excellent level of selectivity of all ligands with respect to duplex DNA, as evidenced by negligible stabilization of the hairpin $h p 2\left(\Delta T_{\mathrm{m}} \leq 2{ }^{\circ} \mathrm{C}\right)$. In addition, almost no drop of ligand-induced stabilization was observed when fluorescence melting experiments were performed in the presence of excess double-stranded competitor (selfcomplementary 26-mer ds26), and only in the case of Pu24T, the addition of the duplex resulted in decrease of the stabilization induced by ligands $5 a$ and 5 b by $2-3{ }^{\circ} \mathrm{C}$ (Fig. S4 + ). This observation agrees with the results of DCC pull-down experiments, which also indicated moderate selectivity of A2L1-A5 for Pu24T with respect to duplex DNA, as non-negligible amounts of this ligand were pulled-down with $d T_{22}$ and $h p 2$ (cf. Fig. 4).

\section{Conclusions}

In this work, we developed a DCC approach based on the formation of DCLs of acylhydrazones in the conditions compatible with native structures of G4-DNA, followed by identification of putative best binders through a pull-down with biotinylated oligonucleotides immobilized on streptavidincoated magnetic beads. This approach was validated using DCL1, where the ranking of pulled-down ligands was found in a good agreement with their G4-binding properties. Subsequently, we constructed two novel libraries, DCL2 and DCL3, which pointed out to several novel bis(acylhydrazones), including A2-L1-A5, A2-L4-A5, and A2-L4-A2, as promising G4DNA binders. Due to hard synthetic accessibility of nonsymmetric bis(acylhydrazones), compound A2-L1-A5 was emulated through two isomeric carboxamide/acylhydrazone hybrids 5a and 5b. Both hybrids, as well as the bis(acylhydrazone) A2-L4-A2, indeed demonstrated fair affinity to various G4-DNA substrates (with $K_{\mathrm{d}}$ values in the $10-650 \mathrm{nM}$ range) and a good level of G4-vs.-duplex selectivity, as evidenced by isothermal fluorimetric titrations, mass-spectrometric and fluorescence-melting experiments, but failed to outperform the prototype ligand A2-L1-A2. At the current stage, we were not able to obtain ligands with significant selectivity towards one or another G4 topology or another structural feature. Development of such ligands would probably require generation of DCLs from building blocks featuring higher structural diversity and/or more complex structural motifs (e.g., peptide derivatives) as well as optimized pull-down protocols. Nonetheless, our approach resulted in hybrid ligands (5a and $\mathbf{5 b}$ ) whose fair G4 affinity combines with favourable drug-like properties, as illustrated by the values of the corresponding physico-chemical descriptors (e.g., fraction of $\mathrm{sp}^{3}$-hybridized carbon atoms $F\left(\mathrm{sp}^{3}\right.$ ) $=0.21, \operatorname{clog} P=-1.3$ and -1.7 , respectively, versus $F\left(\mathrm{sp}^{3}\right)=$ 0.07, $\operatorname{cog} P=-7.1$ in the case of A2-L2-A2). Thus, nonsymmetric ligands combining an $\mathrm{N}$-methylquinolinium moiety with another aromatic moiety represent an interesting motif for the development of other biologically active G4-DNA binders.

The quest for novel G4 ligands with favourable physicochemical properties is a major challenge in the therapeutic targeting of G4-mediated regulation in living systems. The main advantages of DCC, compared to traditional combinatorial chemistry approaches, reside in rapid generation of large, structurally diverse libraries of putative ligands, as well as in facilitation of the identification of most promising ligands. In this context, this work represents a contribution to the field of DNA targeting by laying a foundation for the future use of acylhydrazone-based libraries for targeting $\mathrm{G} 4$ or other structural motifs formed by nucleic acids.

\section{Conflicts of interest}

There are no conflicts to declare.

\section{Acknowledgements}


The authors thank Dr. Marie-Paule Teulade-Fichou (Institut Curie) for a sample of $\mathrm{PhenDC}_{3}$ and helpful discussions. Dr. Jorge González García and Ms. Hang Kang are acknowledged for the preliminary experiments related to this project, and Dr. Eric Largy (ARNA) and Dr. Frédéric Rosu (IECB) for assistance and discussion regarding mass-spectrometry experiments. The project benefited from access to the Plateforme de BioPhysicoChimie Structurale of the IECB (Univ. Bordeaux, CNRS UMS3033, Inserm US001) for native mass spectrometry. This work was supported by Agence Nationale de la Recherche (grant-in aid ANR-17-CE07-0004-01 to AG), French Ministry of Higher Education, Research and Innovation (PhD fellowship to $\mathrm{OR})$, and Institut Curie.

\section{Notes and references}

¥ Note that, while $\mathbf{A} \mathbf{1}$ and $\mathbf{A} \mathbf{2}$ are shown in the aldehyde form, both aldehydes are fully hydrated in aqueous solutions.

1 J. Spiegel, S. Adhikari and S. Balasubramanian, Trends Chem., 2020, 2, 123-136.

2 D. Varshney, J. Spiegel, K. Zyner, D. Tannahill and S. Balasubramanian, Nat. Rev. Mol. Cell Biol., 2020, 21, 459-474.

3 S. A. Ohnmacht and S. Neidle, Bioorg. Med. Chem. Lett., 2014, 24, 2602-2612.

4 S. Neidle, Nat. Rev. Chem., 2017, 1, 1-10.

5 S. Neidle, J. Med. Chem., 2016, 59, 5987-6011.

6 V. S. Chambers, G. Marsico, J. M. Boutell, M. Di Antonio, G. P. Smith and S. Balasubramanian, Nat. Biotechnol., 2015, 33, 877881.

7 S. P. P. Pany, P. Bommisetti, K. V. Diveshkumar and P. I. Pradeepkumar, Org. Biomol. Chem., 2016, 14, 5779-5793.

8 S. Asamitsu, T. Bando and H. Sugiyama, Chem. Eur. J., 2019, 25, 417-430.

9 M. Garavís, B. López-Méndez, A. Somoza, J. Oyarzabal, C. Dalvit, A. Villasante, R. Campos-Olivas and C. González, ACS Chem. Biol., 2014, 9, 1559-66.

10 M. Tassinari, A. Lena, E. Butovskaya, V. Pirota, M. Nadai, M Freccero, F. Doria and S. Richter, Molecules, 2018, 23, 1874.

11 K. M. Felsenstein, L. B. Saunders, J. K. Simmons, E. Leon, D. R. Calabrese, S. Zhang, A. Michalowski, P. Gareiss, B. A. Mock, J. S. Schneekloth and J. S. Schneekloth, Jr., ACS Chem. Biol., 2015, 11, 151013220852007

12 M. Di Antonio, G. Biffi, A. Mariani, E. A. Raiber, R. Rodriguez and S. Balasubramanian, Angew. Chem. Int. Ed., 2012, 51, 1107311078.

13 K. C. Liu, K. Röder, C. Mayer, S. Adhikari, D. J. Wales and S. Balasubramanian, J. Am. Chem. Soc., 2020, 142, 8367-8373.

14 A. Minard, D. Morgan, F. Raguseo, A. Di Porzio, D. Liano, A. G. Jamieson and M. Di Antonio, Chem. Commun., 2020, 56, 89408943.

15 S. Ladame, Org. Biomol. Chem., 2008, 6, 219-226.

16 P. Frei, R. Hevey and B. Ernst, Chem. Eur. J., 2019, 25, 60-73.

17 O. Ramström and J.-M. Lehn, Nat. Rev. Drug Discov., 2002, 1, 26-36.

18 M. Mondal and A. K. H. Hirsch, Chem. Soc. Rev., 2015, 44, 24552488.
19 A. M. Hartman, R. M. Gierse and A. K. H. Hirsch, European J. Org. Chem., 2019, 2019, 3581-3590.

20 C. R. S. Durai and M. M. Harding, Aust. J. Chem., 2011, 64, 671680.

21 B. L. Miller, Top. Curr. Chem., 2011, 322, 107-137.

22 A. M. Whitney, S. Ladame and S. Balasubramanian, Angew. Chem. Int. Ed., 2004, 43, 1143-1146.

23 S. Ladame, A. M. Whitney and S. Balasubramanian, Angew. Chem. Int. Ed., 2005, 44, 5736-5739.

24 A. Bugaut, K. Jantos, J.-L. Wietor, R. Rodriguez, J. K. M. Sanders and S. Balasubramanian, Angew. Chem. Int. Ed., 2008, 47, 26772680.

25 M. C. Nielsen and T. Ulven, Chem. Eur. J., 2008, 14, 9487-9490.

26 S. Jana, D. Panda, P. Saha, G. D. Pantoş and J. Dash, J. Med. Chem., 2019, 62, 762-773.

27 O. Reznichenko, A. Quillévéré, R. P. Martins, N. Loaëc, H. Kang, M. J. Lista, C. Beauvineau, J. González-García, R. Guillot, C. Voisset, C. Daskalogianni, R. Fåhraeus, M.-P. Teulade-Fichou, M. Blondel and A. Granzhan, Eur. J. Med. Chem., 2019, 178, 13-29.

28 G. Pennarun, C. Granotier, L. R. Gauthier, D. Gomez, F. Hoffschir, E. Mandine, J.-F. Riou, J.-L. Mergny, P. Mailliet and F. D. Boussin, Oncogene, 2005, 24, 2917-2928.

29 A. De Cian, E. DeLemos, J.-L. Mergny, M.-P. Teulade-Fichou and D. Monchaud, J. Am. Chem. Soc., 2007, 129, 1856-1857.

30 E. T. Kool, P. Crisalli and K. M. Chan, Org. Lett., 2014, 16, 14541457.

31 D. Larsen, M. Pittelkow, S. Karmakar and E. T. Kool, Org. Lett., 2015, 17, 274-277.

32 V. T. Bhat, A. M. Caniard, T. Luksch, R. Brenk, D. J. Campopiano and M. F. Greaney, Nat. Chem., 2010, 2, 490-497.

33 E. Bartolami, Y. Bessin, V. Gervais, P. Dumy and S. Ulrich, Angew. Chem. Int. Ed., 2015, 54, 10183-10187.

34 E. Bartolami, Y. Bessin, N. Bettache, M. Gary-Bobo, M. Garcia, P. Dumy and S. Ulrich, Org. Biomol. Chem., 2015, 13, 9427-9438.

35 A. J. Clipson, V. T. Bhat, I. McNae, A. M. Caniard, D. J. Campopiano and M. F. Greaney, Chem. Eur. J., 2012, 18, 1056210570.

36 A. M. Hartman, W. A. M. Elgaher, N. Hertrich, S. A. Andrei, C. Ottmann and A. K. H. Hirsch, ACS Med. Chem. Lett., 2020, 11, 1041-1046.

37 M. C. Miller, R. Buscaglia, J. B. Chaires, A. N. Lane and J. O. Trent, J. Am. Chem. Soc., 2010, 132, 17105-17107.

38 B. Heddi and A. T. Phan, J. Am. Chem. Soc., 2011, 133, 98249833.

39 P. Frei, L. Pang, M. Silbermann, D. Eriş, T. Mühlethaler, O. Schwardt and B. Ernst, Chem. Eur. J., 2017, 23, 11570-11577.

40 A. T. Phan, V. Kuryavyi, H. Y. Gaw and D. J. Patel, Nat. Chem. Biol., 2005, 1, 167-173.

41 W. J. Chung, B. Heddi, F. Hamon, M.-P. Teulade-Fichou and A. T. Phan, Angew. Chem. Int. Ed., 2014, 53, 999-1002.

42 D. D. Le, M. Di Antonio, L. K. M. Chan and S. Balasubramanian, Chem. Commun., 2015, 51, 8048-8050.

43 R. N. Das, M. Andréasson, R. Kumar and E. Chorell, Chem. Sci., 2020, 10529-10537.

44 L. Bonnat, L. Bar, B. Génnaro, H. Bonnet, O. Jarjayes, F. Thomas, J. Dejeu, E. Defrancq and T. Lavergne, Chem. Eur. J., 2017, 23, 5602-5613. 
45 F. Rosu, E. De Pauw and V. Gabelica, Biochimie, 2008, 90, 10741087.

46 A. Marchand, A. Granzhan, K. lida, Y. Tsushima, Y. Ma, K. Nagasawa, M. P. Teulade-Fichou and V. V. Gabelica, J. Am.
Chem. Soc., 2015, 137, 750-756.

47 A. De Rache and J.-L. Mergny, Biochimie, 2015, 115, 194-202.

48 A. Marchand, F. Rosu, R. Zenobi and V. Gabelica, J. Am. Chem. Soc., 2018, 140, 12553-12565.

\section{TOC entry}

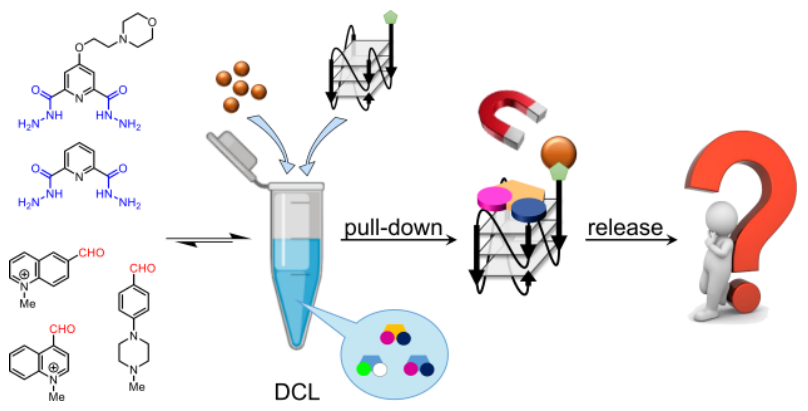

Synthesis of dynamic combinatorial libraries of acylhydrazones in the presence of a G-quadruplex DNA template, followed by pull-down with streptavidin-coated magnetic beads, allows the identification of putative G-quadruplex binders. 